\title{
ON A THEOREM OF C. POSSE CONCERNING GAUSSIAN QUADRATURE OF CHEBYSHEV TYPE
}

\author{
KLAUS-JÜRGEN FÖRSTER
}

\begin{abstract}
We consider $(n, m)$ Chebyshev formulae of algebraic degree $m$ using $n$ nodes. The aim of this short note is to show that by a simple algebraic method C. Posse's theorem concerning Gaussian quadrature of Chebyshev type can be improved. Furthermore, we given an application of this method to Gauss-Kronrod quadrature of Chebyshev type.
\end{abstract}

\section{INTRODUCTION AND STATEMENT OF THE MAIN RESULT}

Let $L$ be a (bounded) linear functional on $C[a, b]$. We say that $L$ admits an $(n, m)$ Chebyshev formula if there are $n$ nodes $x_{\nu, n}^{C} \in \mathbb{R}$ such that

$$
L\left[p_{\mu}\right]=\frac{L\left[p_{0}\right]}{n} \sum_{\nu=1}^{n}\left(x_{\nu, n}^{C}\right)^{\mu} \quad \text { for } \mu=0,1, \ldots, m,
$$

where, here and in the following, $p_{\mu}$ denotes the monomial $p_{\mu}(x)=x^{\mu}$.

Chebyshev formulae have been investigated for more than a hundred years (see, e.g., [6] and the references cited therein). They were first considered by Chebyshev [3], who showed that the linear functionals $T_{\alpha, \beta, \gamma}$,

$$
T_{\alpha, \beta, \gamma}[f]:=\alpha \int_{\beta}^{\gamma} \frac{f(x)}{\sqrt{|x-\beta||x-\gamma|}} d x, \quad \alpha, \beta, \gamma \in \mathbb{R},
$$

admit $(n, 2 n-1)$ Chebyshev formulae for each $n \in \mathbb{N}$, i.e., that each Gaussian quadrature formula is of Chebyshev type. Let us additionally note that the linear functionals $S_{\eta, \xi}$,

$$
S_{\eta, \xi}[f]:=\eta f(\xi), \quad \eta, \xi \in \mathbb{R},
$$

trivially admit $(n, m)$ Chebyshev formulae for all $n, m \in \mathbb{N}$. By a result of Posse [11], $T_{\alpha, \beta, \gamma}$ and $S_{\eta, \xi}$ are the only linear functionals on $C[a, b]$ admitting $(n, 2 n-1)$ Chebyshev formulae for each $n \in \mathbb{N}$. Recently, using methods of complex analysis and Faber polynomials, Peherstorfer [8] has proved the surprising result, that $T_{\alpha, \beta, \gamma}$ and $S_{\eta, \xi}$ also are the only (positive) linear functionals on $C[a, b]$, admitting $(1,1)$ and $(n, n+1)$ Chebyshev formulae for each $n \in \mathbb{N} \backslash\{1\}$. For other improvements of Posse's result see, e.g., [6, 5, 9].

Received by the editor November 6, 1992 and, in revised form, March 24, 1993.

1991 Mathematics Subject Classification. Primary 65D30, 41A55.

Key words and phrases. Chebyshev quadrature, Gaussian quadrature, Gauss-Kronrod quadrature. 
The aim of this note is to show that by a simple algebraic method, introduced by Radau [12] and extended, e.g., in [2, 1, 4], we can obtain more general results. This method is based on Newton's identities (see, e.g., [10, p. $150 \mathrm{ff}]$ ), which, for $n$ arbitrary complex numbers $z_{\nu}$, yield

$$
\left\{\begin{array}{l}
s_{1}+a_{1}=0 \\
s_{2}+a_{1} s_{1}+2 a_{2}=0 \\
\cdots \cdots \\
s_{n-1}+a_{1} s_{n-2}+\cdots+a_{n-2} s_{1}+(n-1) a_{n-1}=0, \\
s_{\lambda+n}+a_{1} s_{\lambda+n-1}+\cdots+a_{n-1} s_{\lambda+1}+a_{n} s_{\lambda}=0 \quad(\lambda=0,1,2, \ldots),
\end{array}\right.
$$

where

$$
\begin{gathered}
s_{\mu}=\sum_{\nu=1}^{n} z_{\nu}^{\mu} \quad(\mu=0,1,2, \ldots), \\
F(t)=\left(t-z_{1}\right)\left(t-z_{2}\right) \cdots\left(t-z_{n}\right)=t^{n}+a_{1} t^{n-1}+\cdots+a_{n-1} t+a_{n} .
\end{gathered}
$$

Theorem. Let $\left(n_{i}\right)_{i=1}^{\infty}$ be a strictly increasing sequence of natural numbers. Let $L$ and $H$ be linear functionals on $C[a, b]$, both admitting $\left(n_{i}, n_{i+1}\right)$ Chebyshev formulae for each $i \in \mathbb{N}$. If

$$
L\left[p_{\mu}\right]=H\left[p_{\mu}\right] \text { for } \mu=0,1, \ldots, n_{1},
$$

then the identity $L=H$ follows.

Proof. First, let $L\left[p_{0}\right] \neq 0$. Applying Newton's identities to (1), we get for $i=1,2, \ldots$

$$
\sum_{\nu=1}^{n_{i}}\left(x_{\nu, n_{i}}^{C}\right)^{\mu}=\frac{n_{i}}{L\left[p_{0}\right]} L\left[p_{\mu}\right] \text { for } \mu=0,1, \ldots, n_{i+1} .
$$

For given $L\left[p_{0}\right], L\left[p_{1}\right], \ldots, L\left[p_{n_{i}}\right]$ we directly obtain that the values of $L\left[p_{\mu}\right]$ are uniquely determined for each $\mu \in\left\{0,1,2, \ldots, n_{i+1}\right\}$. Using (6), we have $L\left[p_{\mu}\right]=H\left[p_{\mu}\right]$ for each $\mu \in \mathbb{N}_{0}$. Since $L$ and $H$ are bounded, by the approximation theorem of Weierstrass the result follows. If $L\left[p_{0}\right]=0$, then with (1) we have $L=H \equiv 0$.

The following corollary extends the results on Chebyshev formulae mentioned in the introduction.

Corollary 1. Let $\left(n_{i}\right)_{i=1}^{\infty}$ be a strictly increasing sequence of natural numbers. Let $L$ be a linear functional on $C[a, b]$ admitting $\left(n_{i}, n_{i+1}\right)$ Chebyshev formulae for each $i \in \mathbb{N}$. Then there exist $\eta, \xi \in \mathbb{R}$ or $\alpha, \beta, \gamma \in \mathbb{R}$, such that, using the notations in (2) and (3),

(i) $L=S_{\eta, \xi}$ if $n_{1}=1$,

(ii) $L=S_{\eta, \xi}$ or $L=T_{\alpha, \beta, \gamma}$ if $n_{1}=2$ and $n_{i+1}<2 n_{i}$ for each $i \in \mathbb{N}$.

Proof. As in the proof of the theorem, we can assume that $L\left[p_{0}\right] \neq 0$. First, let $L\left[p_{2}\right] L\left[p_{0}\right]=L^{2}\left[p_{1}\right]$. We define

$$
H=S_{\eta, \xi}, \quad \eta=L\left[p_{0}\right], \quad \xi=L\left[p_{1}\right] / L\left[p_{0}\right] .
$$


We have $L\left[p_{\mu}\right]=H\left[p_{\mu}\right]$ for $\mu=0,1,2$; therefore, the result follows from the theorem. Now let $L\left[p_{2}\right] L\left[p_{0}\right]>L^{2}\left[p_{1}\right]$. We define

$$
\begin{aligned}
\bar{H}=T_{\alpha, \beta, \gamma}, \quad \alpha & =\frac{L\left[p_{0}\right]}{\pi}, \quad \beta=\frac{L\left[p_{1}\right]-\delta}{L\left[p_{0}\right]}, \quad \gamma=\frac{L\left[p_{1}\right]+\delta}{L\left[p_{0}\right]}, \\
\delta & =\left[2\left(L\left[p_{2}\right] L\left[p_{0}\right]-L^{2}\left[p_{1}\right]\right)\right]^{1 / 2} .
\end{aligned}
$$

A short calculation, using

$$
\bar{H}[f]=\alpha \int_{-1}^{1} f\left(\frac{\gamma-\beta}{2} y+\frac{\gamma+\beta}{2}\right)\left(1-y^{2}\right)^{-1 / 2} d y,
$$

gives $L\left[p_{\mu}\right]=\bar{H}\left[p_{\mu}\right]$ for $\mu=0,1,2$. Since $\bar{H}$ admits $(n, 2 n-1)$ Chebyshev formulae for each $n \in \mathbb{N}$, the result follows from the theorem. Finally, let $L\left[p_{2}\right] L\left[p_{0}\right]<L^{2}\left[p_{1}\right]$. This inequality is equivalent to the inequality

$$
\left(x_{1,2}^{C}\right)^{2}+\left(x_{2,2}^{C}\right)^{2}<\left(x_{1,2}^{C}+x_{2,2}^{C}\right)^{2} / 2 \text {, }
$$

which is impossible for real numbers.

Remarks. 1. We say that a linear functional $L$ on $C[a, b]$ admits extended $(n, m)$ Chebyshev formulae if $x_{\nu, n}^{C} \in \mathbb{C}, x_{\nu, n}^{C}$ real or complex conjugate, and (1) holds. Newton's identities (4) are valid for $z_{\nu} \in \mathbb{C}$, and therefore the theorem is also valid for extended Chebyshev formulae.

2. In the proof of Corollary 1, we see that (11) is possible if and only if $x_{1,2}^{C}, x_{2,2}^{C} \in \mathbb{C}, x_{1,2}^{C}=u+i v, x_{2,2}^{C}=u-i v$, and $v \neq 0$. We obtain $L\left[p_{1}\right]=u L\left[p_{0}\right], L\left[p_{2}\right]=\left(u^{2}-v^{2}\right) L\left[p_{0}\right]$. Defining $\tilde{f}:=\left(p_{1}-u p_{0}\right)^{2}+v^{2} p_{0} / 2$, we have

$$
L[\tilde{f}]=-\frac{1}{2} v^{2} L\left[p_{0}\right], \quad \tilde{f}>0,
$$

which is impossible if $L\left[p_{0}\right] L$ is a positive functional. Therefore, for positive functionals $L$, Corollary 1 also is valid for extended Chebyshev formulae. For $n_{i}=i+1$ this has been proved in [8].

3. In Corollary 1(ii) the assumption $n_{i+1}<2 n_{i}$ cannot be omitted: consider the functional $\bar{S}_{\eta, \xi_{1}, \xi_{2}}$ defined by $\bar{S}_{\eta, \xi_{1}, \xi_{2}}[f]=\frac{\eta}{2}\left[f\left(\xi_{1}\right)+f\left(\xi_{2}\right)\right]$, which admits $(2 n, m)$ Chebyshev formulae for all $n, m \in \mathbb{N}$.

4. In Corollary 1, we only have considered $n_{1}=1$ or $n_{1}=2$. For $n_{1}>2$, using the method described, we are also able to investigate linear functionals admitting (extended) Chebyshev formulae. The author intends to state such results and further applications in a forthcoming paper.

\section{ApPlication to Chebyshev formulae haVING PREASSIGNED NODES}

The above method is also helpful if some of the nodes $x_{\nu, n}^{C}$ of the Chebyshev formulae are preassigned. As an example, we assume that the linear functional $L$ also admits a Gaussian formula of order $k$; i.e., there exist $k$ nodes $x_{\nu, k}^{G}$ and $k$ weights $a_{\nu, k}^{G} \in \mathbb{R}$ such that

$$
L\left[p_{\mu}\right]=\sum_{\nu=1}^{k} a_{\nu, k}^{G}\left(x_{\nu, k}^{G}\right)^{\mu} \text { for } \mu=0,1, \ldots, 2 k-1 .
$$


If $L\left[p_{0}\right] \neq 0$, then a Gaussian formula of order 1 trivially always exists and is uniquely determined by

$$
x_{1,1}^{G}=L\left[p_{1}\right] / L\left[p_{0}\right] .
$$

If there exists also a $(3,2)$ Chebyshev formula such that $x_{1,1}^{G}$ is one of its nodes, then a short calculation using (13) and (1) shows that

$$
\begin{array}{cl}
x_{1,3}^{C}:=x_{1,1}^{G}, & x_{2,3}^{C}=x_{1,3}^{C}-\sqrt{3 / 2} \psi, \quad x_{3,3}^{C}=x_{1,3}^{C}+\sqrt{3 / 2} \psi, \\
& \psi=\frac{1}{L\left[p_{0}\right]} \sqrt{L\left[p_{0}\right] L\left[p_{2}\right]-L^{2}\left[p_{1}\right]} .
\end{array}
$$

Therefore, it is necessary and sufficient for the existence of such a $(3,2)$ Chebyshev formula that

$$
L\left[p_{0}\right] L\left[p_{2}\right] \geq L^{2}\left[p_{1}\right] .
$$

Furthermore, if this $(3,2)$ Chebyshev formula is also a $(3,3)$ Chebyshev formula, then it follows that

$$
L\left[p_{3}\right]=\frac{L\left[p_{1}\right]}{L^{2}\left[p_{0}\right]}\left(3 L\left[p_{0}\right] L\left[p_{2}\right]-2 L^{2}\left[p_{1}\right]\right) .
$$

Inequality (16) is equivalent to the existence of a $(2,2)$ Chebyshev formula,

$$
x_{1,2}^{C}=x_{1,1}^{G}-\psi, \quad x_{2,2}^{C}=x_{1,1}^{G}+\psi,
$$

where $\psi$ is defined in (15). A further short calculation shows that this $(2,2)$ Chebyshev formula is also a $(2,3)$ Chebyshev formula, i.e., a Gaussian formula of order 2, if and only if (17) additionally holds. Therefore, the existence of a $(3,3)$ Chebyshev formula using the node $x_{1,1}^{G}$ is equivalent to the existence of a $(2,3)$ Chebyshev formula.

Now, one may ask, e.g., if there exists an $(n, m)$ Chebyshev formula having some of the nodes $x_{\nu, k}^{G}$ of a Gaussian formula of order $k$. In this situation, using methods from the theory of orthogonal polynomials, Notaris [7], for positive $L$, has recently proved the following interesting result: If, for each $n \in \mathbb{N}$, there exists a $(2 n+1,3 n+1)$ Chebyshev formula with $\left\{x_{\nu, n}^{G} \mid \nu=1,2, \ldots, n\right\} \subseteq\left\{x_{\nu, 2 n+1}^{C} \mid \nu=1,2, \ldots, 2 n+1\right\}$-i.e., these Chebyshev formulae are so-called Gauss-Kronrod formulae-then $L$ is of type $S_{\eta, \xi}$ being defined in (3). The following Corollary 2 extends this result.

Corollary 2. Let $L$ be a linear functional admitting a $(3,4)$ Chebyshev formula and $(2 n+1,2 n+3)$ Chebyshev formulae for each $n \in \mathbb{N} \backslash\{1\}$. For $L\left[p_{0}\right] \neq 0$, let the node $x_{1,1}^{G}$ of the Gaussian formula of order 1 be a node of the $(3,4)$ Chebyshev formula, and let the two nodes $x_{1,2}^{G}, x_{2,2}^{G}$ of the Gaussian formula of order 2 be nodes of the $(5,7)$ Chebyshev formula. Then, there exist $\eta, \xi \in \mathbb{R}$ such that $L=S_{\eta, \xi}$.

Proof. Let $L\left[p_{0}\right] \neq 0$. The existence of a $(3,3)$ Chebyshev formula using the node $x_{1,1}^{G}$ implies that the Gaussian formula of order 2 is a $(2,3)$ Chebyshev formula; see (17) and (18) above. If there exists a $(5,3)$ Chebyshev formula having nodes $x_{1,5}^{C}:=x_{1,2}^{G}=x_{1,2}^{C}$ and $x_{2,5}^{C}:=x_{2,2}^{G}=x_{2,2}^{C}$, then a short 
calculation using

$$
\begin{aligned}
L\left[p_{\mu}\right] & =\frac{L\left[p_{0}\right]}{5}\left\{\sum_{\nu=1}^{2}\left(x_{\nu, 2}^{C}\right)^{\mu}+\sum_{\nu=3}^{5}\left(x_{\nu, 5}^{C}\right)^{\mu}\right\} \\
& =\frac{L\left[p_{0}\right]}{5}\left\{2 \frac{L\left[p_{\mu}\right]}{L\left[p_{0}\right]}+\sum_{\nu=3}^{5}\left(x_{\nu, 5}^{C}\right)^{\mu}\right\} \text { for } \mu=0,1,2,3
\end{aligned}
$$

shows that $\left\{x_{3,5}^{C}, x_{4,5}^{C}, x_{5,5}^{C}\right\}=\left\{x_{1,3}^{C}, x_{2,3}^{C}, x_{3,3}^{C}\right\}$, and therefore, since this $(5,3)$ Chebyshev formula is also a $(5,4)$ Chebyshev formula, it follows that

$$
L\left[p_{4}\right]=\frac{L\left[p_{0}\right]}{5}\left\{\sum_{\nu=1}^{3}\left(x_{\nu, 3}^{C}\right)^{4}+\sum_{\nu=1}^{2}\left(x_{\nu, 2}^{C}\right)^{4}\right\} .
$$

On the other hand, the existence of a $(3,4)$ Chebyshev formula having a node $x_{1,3}^{C}=x_{1,1}^{G}$ yields the identity

$$
L\left[p_{4}\right]=\frac{L\left[p_{0}\right]}{3} \sum_{\nu=1}^{3}\left(x_{\nu, 3}^{C}\right)^{4} .
$$

By comparing (20) and (21), it follows from(15) and (18) that

$$
L^{2}\left[p_{0}\right] \psi^{2}=L\left[p_{0}\right] L\left[p_{2}\right]-L^{2}\left[p_{1}\right]=0 .
$$

Therefore, all nodes $x_{\nu, 3}^{C}$ and $x_{\nu, 5}^{C}$ are equal to $x_{1,1}^{G}$. Since the $(5,4)$ Chebyshev formula is also a $(5,7)$ Chebyshev formula, we have $L\left[p_{\mu}\right]=S_{\eta, \xi}\left[p_{\mu}\right]$ for $\mu=0,1, \ldots, 7$, where $\eta$ and $\xi$ are given in (8). The result now follows from the theorem. If $L\left[p_{0}\right]=0$, then with (1) we have $L \equiv 0$, which is fulfilled for $S_{\eta, \xi}$ with $\eta=0$.

By Corollary 1 it follows that, if there exists a $(2,3)$ Chebyshev formula and $(2 n+1,2 n+3)$ Chebyshev formulae for each $n \in \mathbb{N}$, then $L$ is of type $S_{\eta, \xi}$ or $T_{\alpha, \beta, \gamma}$. For $(2 n+1,2 n+2)$ Chebyshev formulae, using the above method, we have the following result.

Corollary 3. Let $L$ be a linear functional admitting $(2 n+1,2 n+2)$ Chebyshev formulae for each $n \in \mathbb{N}$. For $L\left[p_{0}\right] \neq 0$ let $x_{1,1}^{G}=L\left[p_{1}\right] / L\left[p_{0}\right]$ be a node of each of these $(2 n+1,2 n+2)$ Chebyshev formulae. Then there exist $\eta, \xi \in \mathbb{R}$ or $\alpha, \beta, \gamma \in \mathbb{R}$ such that $L=S_{\eta, \xi}$ or $L=T_{\alpha, \beta, \gamma}$.

Proof. Let $L\left[p_{0}\right] \neq 0$. Since $L$ admits a $(3,2)$ Chebyshev formula having the node $x_{1,3}^{C}:=x_{1,1}^{G}$, the relations (15) imply that $L\left[p_{0}\right] L\left[p_{2}\right] \geq L^{2}\left[p_{1}\right]$. Therefore, there exists a functional $H$ of type $S_{\eta, \xi}$ or $T_{\alpha, \beta, \gamma}$ such that $H\left[p_{\mu}\right]=$ $L\left[p_{\mu}\right]$ for $\mu=0,1,2$-see the proof of Corollary 1 . Now assume that, for given $n \in \mathbb{N}$,

$$
H\left[p_{\mu}\right]=L\left[p_{\mu}\right] \text { for } \mu=0,1, \ldots, 2 n .
$$

In the following, by $x_{\nu, n}^{C H}$ we denote the nodes of the $(n, 2 n-1)$ Chebyshev formula of $H$. Using (1), we have

$$
\sum_{\nu=1}^{2 n+1}\left(x_{\nu, 2 n+1}^{C}\right)^{\mu}=(2 n+1) \frac{L\left[p_{\mu}\right]}{L\left[p_{0}\right]} \text { for } \mu=0,1, \ldots, 2 n+2 .
$$


From (23) it follows that the nodes $x_{1,2 n+1}^{C}, x_{2,2 n+1}^{C}, \ldots, x_{2 n+1,2 n+1}^{C}$ are also the nodes of a $(2 n+1,2 n)$ Chebyshev formula of $H$. Let $F_{2 n+1}^{C}$ and $F_{2 n+1}^{C H}$ be the polynomials

$$
F_{2 n+1}^{C}(t)=\prod_{\nu=1}^{2 n+1}\left(t-x_{\nu, 2 n+1}^{C}\right), \quad F_{2 n+1}^{C H}(t)=\prod_{\nu=1}^{2 n+1}\left(t-x_{\nu, 2 n+1}^{C H}\right) .
$$

Newton's identities (4) and (5) now show that $F_{2 n+1}^{C}(t)-F_{2 n+1}^{C H}(t)$ is equal to a fixed constant $c$ for all $t \in \mathbb{R}$. Since $x_{1,1}^{G}$ is a zero of $F_{2 n+1}^{C H}$ and since $x_{1,1}^{G}$ is also a zero of $F_{2 n+1}^{C}$, it follows that $F_{2 n+1}^{C} \equiv F_{2 n+1}^{C H}$. Therefore, (24) yields $H\left[p_{\mu}\right]=L\left[p_{\mu}\right]$ for $\mu=0,1,2, \ldots, 2 n+2$. Now, by induction, we have $H\left[p_{\mu}\right]=L\left[p_{\mu}\right]$ for each $\mu \in \mathbb{N}$, which, using the approximation theorem of Weierstrass, yields $L=H$.

\section{BIBLIOGRAPHY}

1. L. A. Anderson and W. Gautschi, Optimal weighted Chebyshev type quadrature formulas, Calcolo 12 (1975), 211-248.

2. R. P. Bailey, Convergence of sequences of positive linear functional operations, Duke Math. J. 2 (1936), 287-303.

3. P. L. Chebyshev, Sur les quadratures, J. Math. Pures Appl. (2) 19 (1874), 19-34.

4. K.-J. Förster and G. P. Ostermeyer, On weighted Chebyshev-type quadrature formulas, Math. Comp. 46 (1986), 591-599.

5. K.-J. Förster, On the minimal variance of quadrature formulas, Numerical Integration III (H. Braß and G. Hämmerlin, eds.), Birkhäuser, Basel, 1988, pp. 37-48.

6. W. Gautschi, Advances in Chebyshev quadrature, Numerical Analysis (G. A. Watson, ed.), Lecture Notes in Math., vol. 506, Springer-Verlag, Berlin and New York, 1977, pp. 100-121.

7. S. E. Notaris, On Gauss-Kronrod quadrature formulae of Chebyshev type, Math. Comp. 58 (1992), 745-753.

8. F. Peherstorfer, On Tchebycheff quadrature formulas, Numerical Integration III (H. Braß and G. Hämmerlin, eds.), Birkhäuser, Basel, 1988, pp. 172-186.

9. __ Gauss-Tschebycheff quadratures formulas, Numer. Math. 58 (1990), 273-281.

10. O. Perron, Algebra, de Gruyter, Berlin, 1932.

11. C. Posse, Sur les quadratures, Nouv. Ann. Math. (2) 14 (1875), 49-62.

12. R. Radau, Sur les formules de quadrature à coefficients égaux, C.R. Acad. Sci. Paris 90 (1880), 520-523.

Institut für Mathematik, Universität Hildesheim, 31141 Hildesheim, Germany 